\title{
Characterization of the Recombinant Forms Arising from a Potato virus $X$ Chimeric Virus Infection under RNA Silencing Pressure
}

\author{
D. Barajas, F. Tenllado, and J. R. Díaz-Ruíz \\ Departamento de Biología de Plantas, Centro de Investigaciones Biológicas, CSIC, Campus de la Ciudad Universitaria, \\ Av. Ramiro de Maeztu 9, 28040 Madrid, Spain
}

Submitted 3 February 2006. Accepted 20 March 2006.

\begin{abstract}
Recombination is a frequent phenomenon in RNA viruses whose net result is largely influenced by selective pressures. RNA silencing in plants acts as a defense mechanism against viruses and can be used to engineer virus resistance. Here, we have investigated the influence of RNA silencing as a selective pressure to favor recombinants of PVX-HCT, a chimeric Potato virus $X$ (PVX) vector carrying the helper-component proteinase (HC-Pro) gene from Plum pox virus (PPV). All the plants from two lines expressing a silenced HC-Pro transgene were completely resistant to PPV. However a significant proportion became infected with PVXHCT. Analysis of viral RNAs accumulating in silenced plants revealed that PVX-HCT escaped silencing-based resistance by removal of the HC-Pro sequences that represented preferential targets for transgene-promoted silencing. The virus vector also tended to lose the HC-Pro insert when infecting transgenic plants containing a nonsilenced HC-Pro transgene or wild-type (wt) Nicotiana benthamiana plants. Nevertheless, loss of HC-Pro sequences was faster in nonsilenced transgenic plants than in wt plants, suggesting the transgene plays a role in promoting a higher selective pressure in favor of recombinant virus versions. These results indicate that the outcome of recombination processes depends on the strength of selection pressures applied to the virus.
\end{abstract}

Additional keywords: HC-Pro transgenic plants, virus stability, siRNA.

RNA silencing is an RNA-based regulatory mechanism conserved in most eukaryotes that plays fundamental roles in the regulation of gene expression, epigenetic DNA modifications, and defense against viruses and transposable elements (Baulcombe 2004; Zamore and Haley 2005). In viral defense, the RNA silencing machinery recognizes several features of viral infections involving the formation of double-stranded (ds) RNA and initiates a process leading to the degradation of viral RNAs (Szittya et al. 2002; Voinnet 2005). In this process, dsRNA is cleaved by dicer enzymes into short-interfering (si) RNA molecules (21 to 26 nucleotides in length) that guide the degradation of cognate RNAs. In productive viral infections, most viruses can counteract the RNA silencing defense, in part

Corresponding author: J. R. Díaz-Ruíz; E-mail: jrdiazruiz@cib.csic.es

* The $\boldsymbol{e}$-Xtra logo stands for "electronic extra" and indicates that Figure 1 appears in color online and the HTML abstract available on-line contains supplemental material not included in the print edition. by expressing proteins that interfere with the RNA-silencing machinery (Moissiard and Voinnet 2004; Silhavy and Burgyan 2004). Some of these viral proteins, such as the helper-component proteinase (HC-Pro) from potyviruses, can interfere with the RNA-silencing effector step, leading to inhibition of siRNA-guided RNA degradation (Chapman et al. 2004; Dunoyer et al. 2004). In addition to its natural role in viral defense, RNA silencing can also be used to engineer viral resistance in plants by expressing transgenes containing virusderived sequences (Beachy 1997; Prins 2003). Silencing of the transgene would target viral RNAs containing enough sequence similarity to the transgene for degradation, therefore inhibiting virus infection (Barajas et al. 2004; Tenllado and Díaz-Ruíz 1999). This strategy has been used to engineer resistance against many viruses in a number of plant species and is likely to be effective against almost any kind of plant virus (Beachy 1997; Llave et al. 2004; Tenllado et al. 2004).

Virus vectors have been developed from a variety of plant viruses and are useful tools for a number of biotechnological applications, including expression of high levels of foreign proteins and silencing of plant genes through virus-induced gene silencing (VIGS) (Burch-Smith et al. 2004; Cañizares et al. 2005; Scholthof et al. 1996). Virus vectors have also been used to investigate some aspects of virus resistance in transgenic plants. The main advantage of using virus vectors is that the foreign sequence inserted is not required for the virus and can be manipulated without affecting virus viability. However, the instability of virus vectors represents a strong disadvantage for some applications, since they tend to remove the inserted sequence during infection, likely as a result of RNA recombination (Chapman et al. 1992; Guo et al. 1998; Rabindran and Dawson 2001).

Recombination is one of the most important mechanisms affecting virus evolution, facilitating the incorporation of advantageous traits and promoting the removal of disadvantageous properties (García-Arenal 2003). RNA recombination is thought to occur most frequently by a copy-choice mechanism during replication, in which the replicase dissociates from the original template and resumes polymerization, using a different RNA or a different part of the same RNA molecule as a template (Nagy and Simon 1997). In RNA viruses, recombination events are frequent and likely contribute to a rapid adaptation of viruses to their hosts (Lai 1992). Foreign inserts are usually a disadvantageous load for viruses, affecting accumulation and spread, and they tend to be removed during infection as a result of selection pressure. In a recent report, however, the propensity of a Tomato bushy stunt virus (TBSV) vector to lose the coding sequence for the green fluorescent protein (GFP) gene was reduced when this 
insert was targeted by RNA silencing (Zhong et al. 2005). Maintenance of the foreign insert was not expected to give a selective advantage to this virus, suggesting that silencing may influence the recombination process; therefore, factors other than selection pressure may influence the outcome of RNA recombination.

In the present study, we analyzed virus resistance and recombination in transgenic Nicotiana benthamiana plants containing a silenced HC-Pro gene from Plum pox virus (PPV). Although these plants were completely resistant to PPV, they showed incomplete resistance to PVX-HCT, a Potato virus X (PVX)based vector carrying the PPV HC-Pro gene. PVX-HCT escaped silencing-based resistance upon removal of the HC-Pro sequences that represented preferential targets for RNA silencing. PVX-HCT also tended to lose the HC-Pro insert in nonsilenced HC-Pro transgenic plants and in wild type (wt) N. benthamiana plants. Furthermore, deletion of HC-Pro sequences occurred faster in nonsilenced transgenic plants than in wt plants, suggesting a role of the HC-Pro transgene in promoting deletion of HC-Pro sequences from the virus. The results indicate that the kind of selection pressure and maybe other RNA silencingrelated processes influence the outcome of RNA recombination.

\section{RESULTS}

Resistance to the chimeric virus PVX-HCT

in silenced transgenic plants.

In a previous study, we reported resistance to PPV in $N$. benthamiana plants transformed with a translatable version of the PPV HC-Pro gene. Two transgenic lines showed resistance to PPV due to RNA silencing induced by the HC-Pro transgene (Barajas et al. 2004). In the present study, we used line HC2.6, a homozygous $\mathrm{R}_{2}$ progeny derived from one of the previously reported lines, to study virus resistance. This line showed complete resistance to PPV in all plants (Table 1), with no virus accumulation detectable by Northern blot analysis in systemic or inoculated leaves (data not shown).

According to the current knowledge of virus resistance based on RNA silencing, we would expect HC-Pro transgenic plants to be resistant to any virus containing enough sequence similarity to the transgene. With this purpose in mind, we tested resistance in line HC2.6 against PVX-HCT, a PVXbased chimeric virus carrying a translatable version of the PPV HC-Pro gene. PVX-based chimeric viruses tend to lose the inserted foreign sequence during infection (Chapman et al. 1992; González-Jara et al. 2005). For this reason, sap inoculum to be used in resistance experiments was prepared from the first systemically infected leaves of PVX-HCT infected plants, in which loss of the HC-Pro insert is less likely. Northern blot analysis of leaves used to prepare PVX-HCT inoculum showed no detectable accumulation of deleted forms of this chimeric virus (Fig. 1A). Wt $N$. benthamiana plants challenged with PVX-HCT sap inoculum showed symptoms of systemic infection by 7 days postinoculation (dpi), followed by the appearance of a typical severe necrosis by 12 to 14 dpi (Fig. 1B) caused by the expression of the HC-Pro protein (González-Jara et al. 2005).

Table 1. Susceptibility of wt Nicotiana benthamiana and different transgenic lines to recombinant Potato virus X (PVX) viruses

\begin{tabular}{|c|c|c|c|c|c|c|}
\hline Inoculum & Virus & N. benthamiana & C2.2 & HC2.20 & HC2.6 & HCn11.20 \\
\hline Sap & $\begin{array}{l}\text { Plum pox virus } \\
\text { PVX-HCT } \\
\text { PVX-HCT }_{(1 / 10)}{ }^{b}\end{array}$ & $\begin{array}{l}15 / 15^{a} \\
11 / 11 \\
6 / 6\end{array}$ & $\begin{array}{l}12 / 12 \\
\ldots \\
\ldots\end{array}$ & $\begin{array}{l}19 / 19 \\
\ldots \\
\ldots\end{array}$ & $\begin{array}{l}0 / 68 \\
19 / 29 \\
4 / 20\end{array}$ & $\begin{array}{l}0 / 69 \\
\ldots \\
\cdots\end{array}$ \\
\hline RNA & $\begin{array}{l}\text { PVX-HCT } \\
\text { PVX-HCL }{ }_{134} \mathrm{H} \\
\text { PVX-HC } \Delta-5 \\
\text { PVX-HC } \Delta-6 \\
\text { PVX-HC } \Delta-10 \\
\text { PVX-HC } \Delta-2 \mathrm{c} \\
\text { PVX-HC-5' } \\
\text { PVX-HC-C } \\
\text { PVX-HC-3' }\end{array}$ & $\begin{array}{l}11 / 12 \\
16 / 16 \\
6 / 6 \\
5 / 6 \\
6 / 6 \\
5 / 6 \\
8 / 8 \\
8 / 8 \\
7 / 8\end{array}$ & $\begin{array}{l}17 / 18 \\
\ldots \\
\ldots \\
\ldots \\
\ldots \\
\ldots \\
\ldots \\
\ldots \\
\ldots\end{array}$ & $\begin{array}{l}17 / 18 \\
\ldots \\
\ldots \\
\ldots \\
\ldots \\
\ldots \\
\ldots \\
\ldots \\
\ldots\end{array}$ & $\begin{array}{l}23 / 95 \\
8 / 39 \\
14 / 20 \\
17 / 20 \\
18 / 20 \\
15 / 20 \\
31 / 34 \\
12 / 34 \\
10 / 34\end{array}$ & $\begin{array}{l}11 / 60 \\
\ldots \\
\ldots \\
\ldots \\
\ldots \\
\ldots \\
\ldots \\
\ldots \\
\ldots\end{array}$ \\
\hline
\end{tabular}

${ }^{a}$ Number of infected plants/number of inoculated plants.

b Sap inoculum diluted 10-fold in $20 \mathrm{mM}$ sodium phosphate buffer.
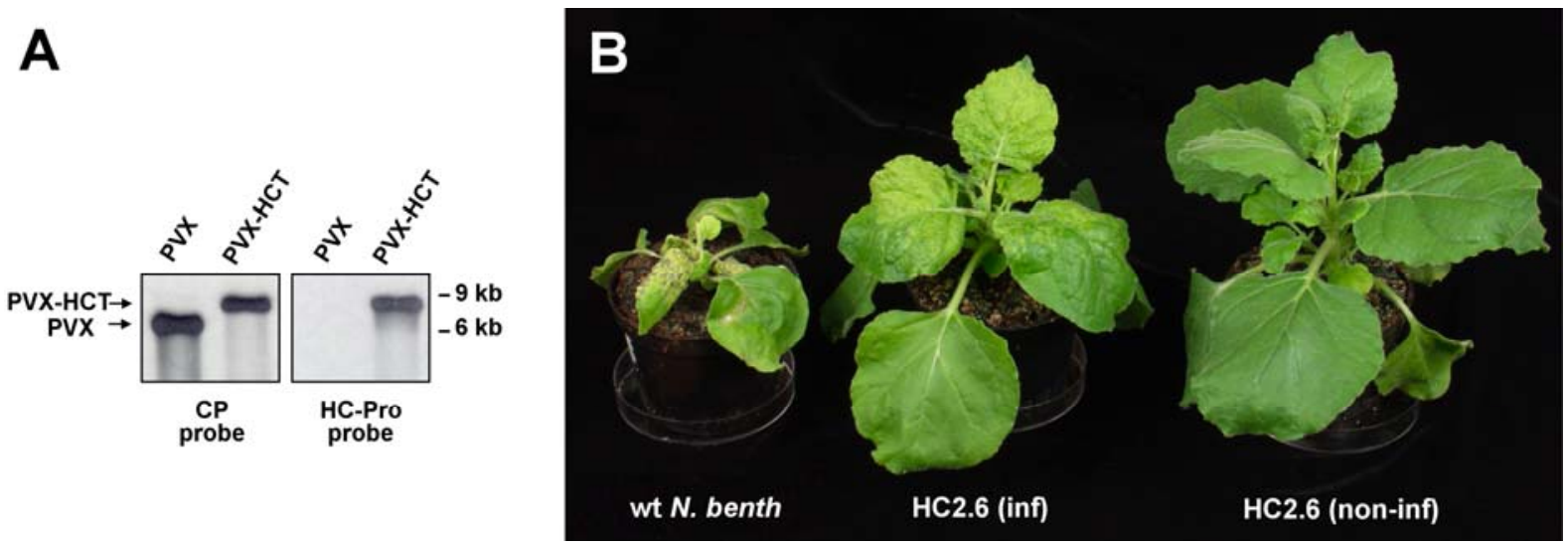

Fig. 1. Analysis of resistance to Potato virus X (PVX)-HCT in helper-component proteinase (HC-Pro)-silenced plants. A, Northern blot analysis of leaf tissue used to prepare PVX-HCT sap inoculum. Viruses were detected with digoxigenin-labeled RNA probes complementary to either the PVX capsid protein (CP) gene sequence or to the Plum pox virus HC-Pro gene sequence, as indicated. The position of RNA markers (kb) is indicated. B, Symptoms caused by PVXHCT at 14 days postinoculation. Wild-type (wt) Nicotiana benthamiana plants developed extensive necrosis promoted by expression of HC-Pro from PVXHCT. HC2.6 plants that became infected (inf) showed a chlorotic mosaic with no extensive necrosis. Some of the HC2.6 plants inoculated with PVX-HCT did not develop-symptoms of infection (noninf). 
Surprisingly, when HC2.6 plants were challenged with PVX-HCT, a high proportion of the plants became infected (65\%; Table 1). These plants started to show systemic symptoms by 7 to 12 dpi. However, they never showed extensive necrosis as observed in wt $N$. benthamiana plants (Fig. 1B). Similar experiments repeated with different sap preparations yielded similar results. The fact that a high proportion of HC2.6 plants became infected could be due to the possibility that the sap inoculum contained low amounts of deleted versions of PVX-HCT that had lost the HC-Pro insert and, hence, were not targeted by the resistance mechanism. To test this hypothesis and avoid possible problems arising from the use of sap inocula, we repeated these experiments using in vitro RNA transcripts of PVX-HCT as inoculum. Wt $N$. benthamiana plants inoculated with PVX-HCT RNA transcripts showed symptoms of systemic infection typically by 7 to $10 \mathrm{dpi}$, followed by the appearance of extensive necrosis around 13 to 16 dpi. Inoculation of either transgenic plants that carried an empty vector lacking the HC-Pro sequence (line C2.2) or nonsilenced HC-Pro transgenic plants (line HC2.20) yielded similar results (Table 1). When HC2.6 plants were inoculated with PVX-HCT RNA transcripts, a significant proportion became infected (24\%; Table 1), displaying systemic symptoms at different times ranging from 9 to $18 \mathrm{dpi}$ but without showing extensive necrosis. These results show that infection of HC2.6 plants with PVX-HCT was not necessarily caused by a hypothetical presence of deleted forms of the virus in the sap inoculum. The lower proportion of infected plants observed with the RNA inoculum compared to the sap inoculum was likely a consequence of a lower infectivity of the RNA inoculum. In this regard, a 10-fold diluted sap inoculum produced a proportion of infected HC2.6 plants comparable to that observed when RNA was used as inoculum (20\%; Table 1).

\section{Role of HC-Pro in overcoming resistance by PVX-HCT.}

In a previous study reporting resistance to PPV in HC-Pro transgenic plants, we showed that resistance was caused by RNA silencing induced by the HC-Pro transgene in spite of encoding a suppressor of RNA silencing (Barajas et al. 2004). Silencing of the HC-Pro transgene was not likely compromised by the HC-Pro protein expressed from the transgene itself, probably because of its low accumulation (below 1/200 the level observed in a PPV infection; Barajas et al. 2004). However, this point is rather controversial, especially after the results shown here with PVX-HCT, and a possible role of $\mathrm{HC}$ Pro expressed from the transgene in assisting PVX-HCT to surpass the resistance mechanism should be considered. To test this, we analyzed resistance to PVX-HCT in the transgenic line $\mathrm{HCn} 11.20$, a homozygous $\mathrm{R}_{2}$ line that carries a nontranslatable version of the HC-Pro transgene and shows complete resistance to PPV (Table 1). When this line was challenged with PVX-HCT RNA transcripts, a number of plants showed symptoms of systemic infection by 9 to 19 dpi without extensive necrosis. Both the proportion of infected plants and the time of appearance of symptoms were similar to those observed in line HC2.6 (18\%; Table 1), suggesting that the HCPro protein expressed in HC2.6 does not contribute significantly to cause the incomplete resistance observed against PVX-HCT.

Alternatively, the HC-Pro protein artificially expressed from PVX-HCT may as well help the virus to surpass the resistance mechanism. To clarify this point, we used the chimeric virus PVX-HCL ${ }_{134} \mathrm{H}$, which carries a PPV HC-Pro gene with a mutation in amino acid 134 that precludes its capability to suppress RNA silencing (González-Jara et al. 2005). HC2.6 plants inoculated with PVX-HCL ${ }_{134} \mathrm{H}$ RNA transcripts yielded a proportion of plants with symptoms of systemic infection $(20 \%$;
Table 1) appearing by 8 to $20 \mathrm{dpi}$, which was similar to the results observed with PVX-HCT. Therefore, these results do not support an influence of HC-Pro, expressed from either the transgene or the chimeric virus, in assisting PVX-HCT to overcome resistance.

\section{Analysis of PVX-HCT accumulating in HC-Pro transgenic plants.}

As mentioned above, resistance in HC2.6 and HCn 11.20 plants would be expected to be effective against any virus carrying enough sequence similarity to the silenced HC-Pro transgene. Thus, the lack of resistance to PVX-HCT in some plants was rather unexpected. To understand what makes PVX-HCT escape the resistance mechanism, we analyzed virus accumulation in systemically infected leaves of RNAinoculated plants. As controls, plants from lines C2.2 (empty vector) and HC2.20 (nonsilenced HC-Pro transgene) were analyzed at $9 \mathrm{dpi}$. At this time, all plants from these lines showed accumulation of PVX-HCT with the expected size for genomic and subgenomic RNAs (Fig. 2B). Plants from lines HC2.6 and HCn11.20 showed symptoms at variable times (typically starting at 9 to $19 \mathrm{dpi}$ ), so samples from each of these plants were taken within two days after the first appearance of symptoms from the second systemically infected leaf. All of these plants showed genomic and subgenomic RNAs of smaller size than those expected for PVX-HCT (Fig. 2C). Considering the foreign origin of the HC-Pro insert and the lack of necrotic symptoms in these plants, the smaller size of viral RNAs was likely caused by deletions affecting the HC-Pro insert. These deletions varied in length among plants, as indicated by the different sizes of genomic and subgenomic RNAs. Some of the plants showed accumulation of virus that had likely lost most of the HC-Pro insert, because viral RNAs were undetectable when using an HCPro probe, and had a size close to that of PVX RNAs (e.g., HC2.6 plants 16, 2b, 4b, and HCn11.20 plant 5c; Fig. 2C). In other plants, viral RNAs were readily detectable with an HCPro probe, with sizes ranging between those of PVX-HCT and PVX RNAs (e.g., HC2.6 plants 6, 9, 10, and 15, HCn 11.20 plants $1 \mathrm{c}, 2 \mathrm{c}, 3 \mathrm{c}, 4 \mathrm{c}$, and $6 \mathrm{c})$. Other plants, such as HC2.6 plant 8, likely contained a mixture of different virus populations, as suggested by the detection of two differentsized genomic RNAs (Fig. 2C). We generically termed these viruses, accumulating in HC2.6 and HCn11.20 plants, as PVX-HC $\Delta$ variants.

Deletions affecting PVX-HC $\Delta$ variants were analyzed by reverse transcriptase-polymerase chain reaction (RT-PCR) with primers spanning a wide area surrounding the HC-Pro insertion site in PVX vector. These reactions yielded products ranging in size between those expected for PVX and PVXHCT amplification products. Most plants showed a single major product, while some clearly showed more than one product (e.g., HC2.6 plants 7 and $3 b$ and HCn11.20 plants $1 c$ and 3c; Fig. 3A). Amplification products were sequenced. For those plants showing more than one product, no clear sequences were obtained; therefore, these amplification products were isolated from the gel and were sequenced separately. Sequences of PVX-HC $\Delta$ variants demonstrated the appearance of deletions affecting the HC-Pro insert (Fig. 3B). Deletions spanned different portions of the HC-Pro sequence, affecting more profusely the central to $3^{\prime}$ area, and the largest area was conserved in the PVX-HC $\Delta$ variant accumulating in HC2.6 plant 10 (PVX-HC $\Delta-10)$. In some cases, deletions affected one of the two duplicated subgenomic promoters (e.g., PVX-HC $\Delta$ plants 3, 7.2, 16, 3b.2, 4b, and 5c; Fig. 3B) but never affected the PVX 8k or capsid protein $(\mathrm{CP})$ coding regions surrounding the HC-Pro insertion site. 


\section{Infectivity of PVX-HC $\Delta$ variants and siRNA abundance} in silenced plants.

Given the above results, deletion of most or all of the HCPro sequence is likely the factor that determines the capability of PVX-HCT to initiate infection in HC2.6 and HCn11.20 plants. To support this hypothesis, we cloned the HC-Pro portions that were retained in some of the PVX-HC $\Delta$ variants into PVX. Cloning these portions newly into PVX eliminates possible minor PVX-HCA variants that may be present in the plants but also permits the production of RNA inocula that would make the results comparable to those obtained with PVX-HCT RNA. We cloned PVX-HC $\Delta-5$, PVX-HC $\Delta-6$, PVX$\mathrm{HC} \Delta-10$, and PVX-HC $\Delta-2 \mathrm{c}$ in this manner. RNA transcripts of these constructs showed an infectivity similar to that observed for PVX-HCT RNA, infecting most of the control wt $N$. benthamiana plants. Inoculation of these viruses onto HC2.6 plants yielded a proportion of infected plants much higher than that observed with PVX-HCT (70 to 90 versus $24 \%$; Table 1). The susceptibility of HC2.6 plants to each of the four cloned PVX-HC $\Delta$ variants was quite similar and independent of the size of the HC-Pro portion retained in each one. These results, therefore, confirm that PVX-HC $\Delta$ variants are less susceptible to the virus-resistance mechanism operating in HC2.6 plants than the original PVX-HCT. The likely scenario is that, although PVX-HCT is targeted by the resistance mechanism operating in HC2.6 and HCn11.20 plants, in some plants, it gives rise to deleted forms that are less efficiently targeted by the resistance mechanism, and consequently, these are capable of infecting the plants systemically.
Surprisingly, some of the deleted virus variants that accumulated in HC2.6 or HCn11.20 plants contained considerable portions of the HC-Pro sequence and still were capable of infecting these plants. The largest portions retained in PVX-HC $\Delta$ variants spanned the $5^{\prime}$ end of the HC-Pro sequence (e.g., PVX-HC $\Delta$ plant 10; Fig. 3B), while retained portions corresponding to the $3^{\prime}$ end were smaller (Fig. 3B). These observations suggested that RNA silencing-based virus resistance in $\mathrm{HC} 2.6$ plants might be targeted more efficiently to the $3^{\prime}$ region of the HC-Pro sequence than to the $5^{\prime}$ region. We analyzed this possibility by separately cloning into PVX three fragments of similar size, collectively spanning the whole HC-Pro sequence. PVX-HC-5' contains the 5' third of the HC-Pro sequence (nucleotides 1 to 443), PVX-HC-C contains the central third (nucleotides 453 to 895), and PVXHC- $3^{\prime}$ contains the $3^{\prime}$ third (nucleotides 866 to 1,374). These constructs were transcribed in vitro and were inoculated onto plants. Infectivity of these viruses in wt $N$. benthamiana was similar to that of other PVX variants. Inoculation of these viruses onto HC2.6 plants yielded different proportions of infected plants (Table 1). PVX-HC-5' infected a high proportion of HC2.6 plants (90\%; Table 1), similar to that observed with PVX-HCA variants. In contrast, PVX-HC-C and PVXHC-3' infected a minor proportion of HC2.6 plants (29 to $35 \%$; Table 1), closer to that observed with PVX-HCT. Therefore, these results strongly argue in favor of the hypothesis that RNA silencing promoted by the HC-Pro transgene may be targeted preferentially against the central and 3' regions of the HC-Pro sequence.
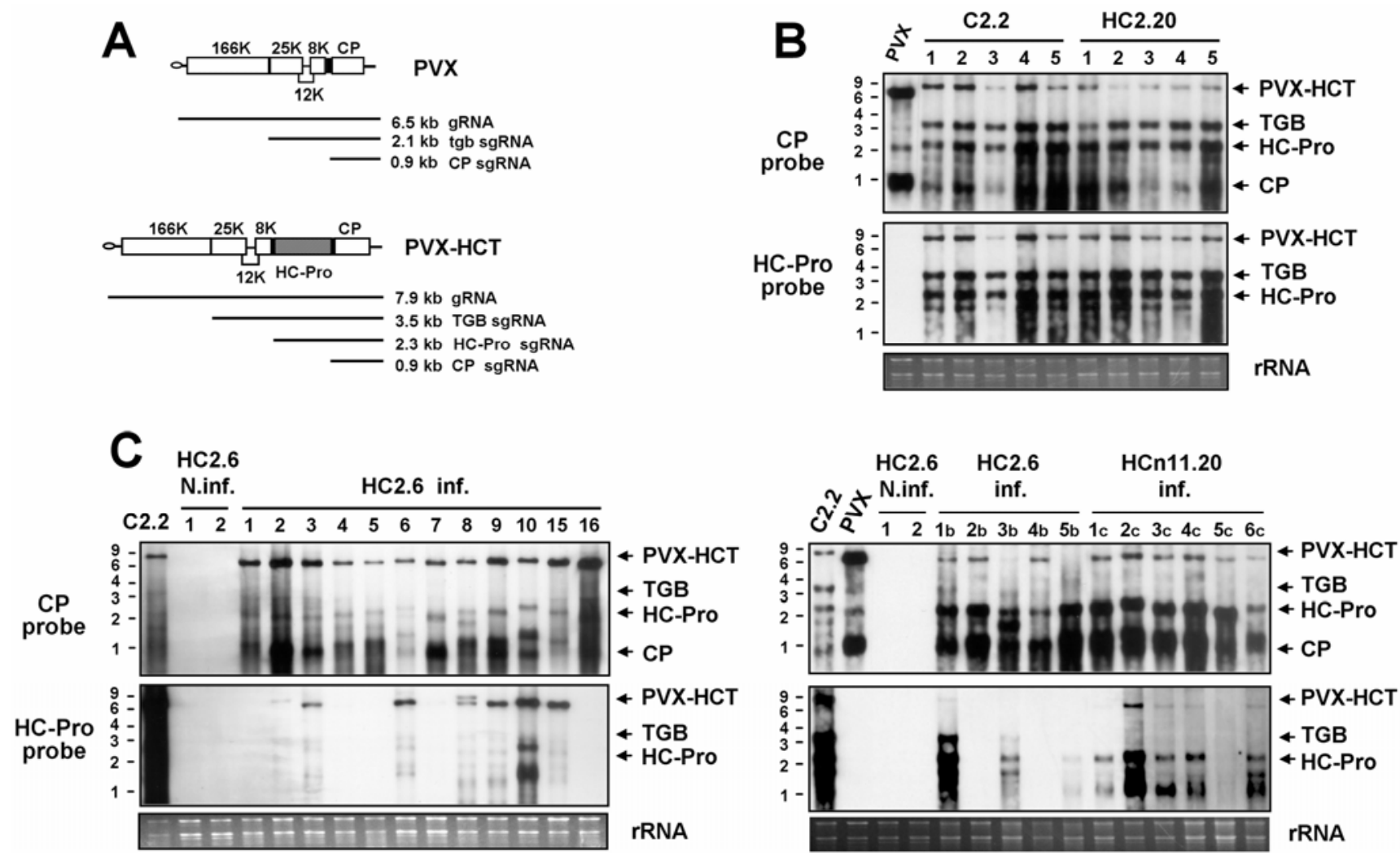

Fig. 2. Analysis of Potato virus $X$ (PVX)-HCT RNAs accumulating in helper-component proteinase (HC-Pro)-silenced plants. A, Genomic organization of PVX and the chimeric virus PVX-HCT and expected sizes of genomic (g) and subgenomic (sg) RNAs promoting translation of the triple gene block genes (TGB), HC-Pro, and capsid protein (CP). B, Analysis of PVX-HCT RNAs at 9 days postinoculation (dpi) in individual plants from lines C2.2 (transformed with empty vector) and HC2.20 (containing a nonsilenced HC-Pro transgene). C, Analysis of PVX-HCT 2 days after appearance of symptoms (9 to 19 dpi) in individual plants from lines HC2.6 and HCn11.20 (containing silenced HC-Pro transgenes) or in HC2.6 plants not showing symptoms (N.inf.). The position of RNA markers $(\mathrm{kb})$ are indicated on the left of each panel. Expected sizes of genomic and subgenomic PVX-HCT RNAs are indicated on the right of each panel. Top panels in B and C show detection with a digoxigenin (DIG)-labeled RNA probe complementary to the PVX CP gene. Middle panels show detection with a DIG-labeled RNA probe complementary to the Plum pox virus HC-Pro gene. Lower panels show RNAs stained with ethidium bromide. 
To confirm this hypothesis, we analyzed the accumulation of siRNAs derived from different regions of the HC-Pro transgene. The HC-Pro transgene in lines HC2.6 and HCn11.20 comprises the PPV HC-Pro sequence and is followed by the $\beta$ glucuronidase (GUS) gene sequence, so that a fusion mRNA is expressed (Barajas et al. 2004). To analyze siRNA accumulation, we used probes corresponding to the same HC-Pro fragments that had been cloned into PVX (HC-5', HC-C, and HC$3^{\prime}$ ), and probes corresponding to either the $5^{\prime}$ or $3^{\prime}$ halves of the GUS sequence (GUS-5' and GUS-3') (Fig. 4A). siRNAs derived from the HC-Pro transgene were detected in HC2.6 and HCn11.20 plants with probes HC-C, HC-3', and GUS-5' (Fig. 4B). In contrast, no siRNAs were detected with probes HC-5' or GUS-3'. To permit a better comparison among the accumulation levels of siRNAs, we isolated and labeled the siRNA fraction from HC2.6 and HCn11.20 plants. These labeled siRNAs were hybridized with equal amounts of DNA corresponding to the five fragments of the $\mathrm{HC}$-Pro transgene. siRNAs from HC2.6 plants reacted clearly with HC-C, HC-3', and GUS-5' fragments but very slightly with $\mathrm{HC}-5^{\prime}$ and GUS$3^{\prime}$ ones (Fig. 4C). siRNAs from HCn11.20 plants also showed a similar pattern (Fig. 4C). These results show that siRNAs were not equally distributed along the HC-Pro transgene sequence, being more abundant around the central region of the transgene. The relative abundance of siRNAs from each portion of the HC-Pro transgene was in agreement with the different susceptibility of HC2.6 plants to PVX-HC-5', PVX-HC-C, and PVX-HC-3' (Table 1). PVX-HC $\Delta$ variants identified in HC2.6 and HCn11.20 plants reflected this unequal siRNA abundance, with virus variants containing longer portions from the $5^{\prime}$ end of the HC-Pro sequence (Fig. 3B). Therefore, PVX-
HC $\Delta$ variants arising from PVX-HCT are likely to proliferate and spread in HC2.6 and HCn11.20 plants once they have lost a critical amount of silencing-targeted sequences.

\section{Analysis of virus stability \\ in HC2.20 and wt $N$. benthamiana plants.}

To better understand the role of RNA silencing in favoring the appearance of PVX-HC $\Delta$ variants in $\mathrm{HC} 2.6$ and $\mathrm{HCn} 11.20$ plants, we analyzed the stability of the HC-Pro insert during infections in wt $N$. benthamiana and in $\mathrm{HC} 2.20$ (nonsilenced HC-Pro transgene) plants. To do so, we used PVX-HCL ${ }_{134} \mathrm{H}$. This chimeric virus encodes an HC-Pro protein impaired in the suppression of RNA silencing and does not produce necrosis in infected plants, allowing sample collection several days postinoculation. We analyzed the stability of the HC-Pro insert by RT-PCR at $9,12,16$, and 20 dpi, taking samples from the second, third, fifth, and seventh systemically infected leaves, respectively. By 9 dpi, both wt $N$. benthamiana and HC2.20 plants yielded amplification products corresponding in size to the original virus, although smaller-sized products denoting the presence of virus variants with deletions in the HC-Pro insert were already detectable (Fig. 5). At later dpi, amplification products of smaller size became predominant in all plants, indicating that virus variants with deletions in the HC-Pro insert were outcompeting the original PVX-HCL ${ }_{134} \mathrm{H}$. By 16 to $20 \mathrm{dpi}$, plants mainly yielded amplification products close in size to PVX vector, indicating loss of most of the HC-Pro insert. Some of the amplification products were smaller than expected for the PVX vector (e.g., N. benthamiana plant 5 or HC2.20 plant 4 at 20 dpi; Fig. 5), suggesting the loss of sequences from one of the duplicated subgenomic promoters. Sequence analysis of these prod-
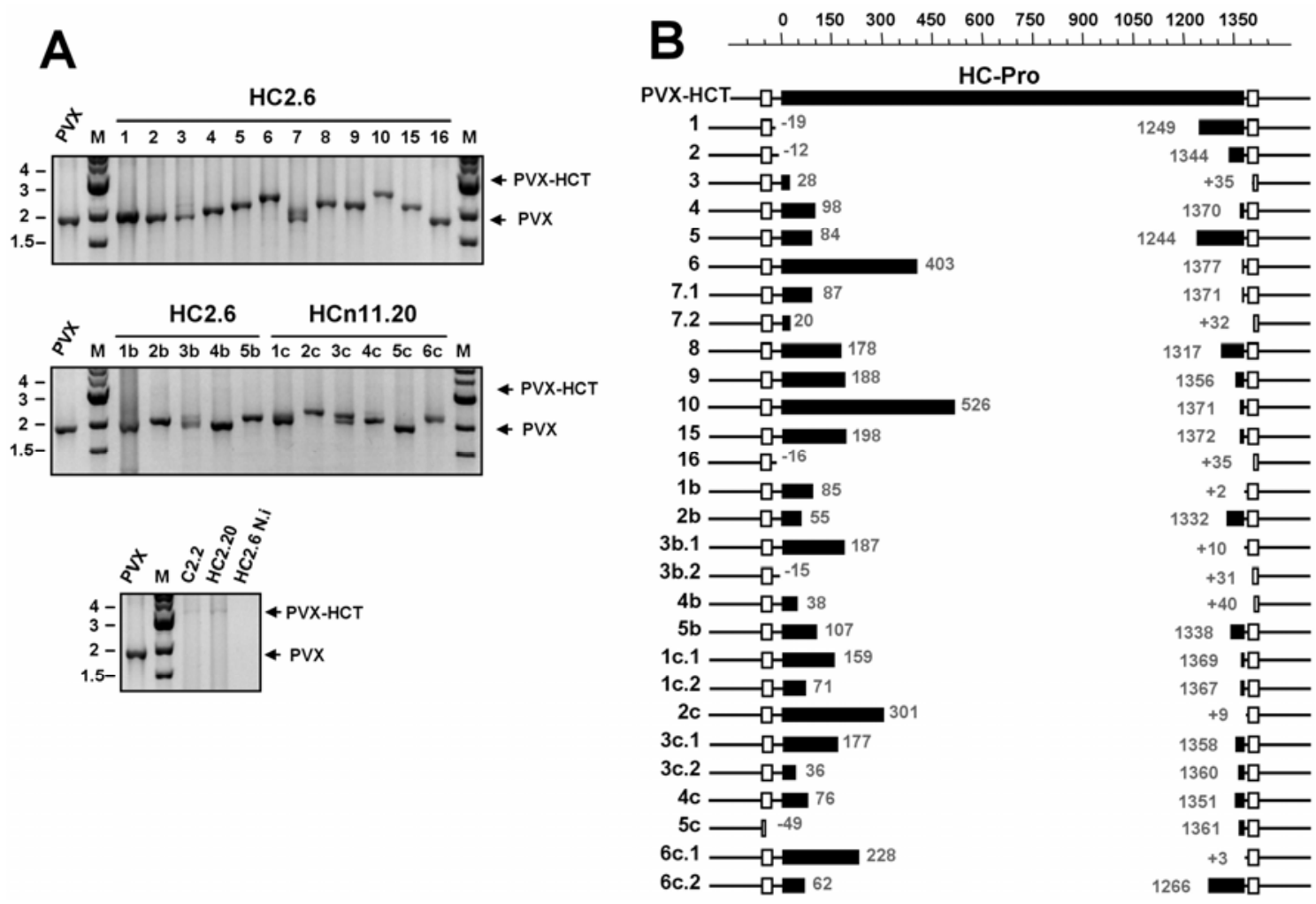

Fig. 3. Analysis of deletions affecting the helper-component proteinase (HC-Pro) insert in Potato virus X (PVX)-HCT. A, Reverse transcriptase-polymerase chain reaction analysis of PVX-HCT in individual HC2.6 and HCn11.20 plants. C2.2 and HC2.20 plants infected with PVX-HCT as well as a noninfected (N.i) HC2.6 plant were used as controls. The expected position for amplification products from PVX and PVX-HCT is indicated on the right of each panel. The positions of DNA markers (M) are indicated on the left $(\mathrm{kb})$. B, Diagram showing the deletions in PVX-HC $\Delta$ forms that accumulated systemically in HC2.6 and HCn11.20 plants. Black boxes represent HC-Pro sequences. White boxes represent the subgenomic promoters, and lines stand for surrounding PVX sequences. The positions of deletions in the HC-Pro nucleotide sequence are indicated. - and + indicate number of nucleotides spanning $5^{\prime}$ and $3^{\prime}$ further from the HC-Pro sequence, respectively. 
ucts confirmed this point. However, this did not involve perfect homologous recombination between identical sequences of the subgenomic promoters (data not shown). The results indicate that virus variants with deletions of the HC-Pro insert appear independently of the silencing status of the plants. Comparison of the results from wt $N$. benthamiana and HC2.20 plants also showed that deleted virus variants outcompeted the original PVX-HCL ${ }_{134} \mathrm{H}$ faster in $\mathrm{HC} 2.20$ plants than in wt $N$. benthamiana plants (Fig. 5). This suggests that the presence of a nonsilenced HC-Pro transgene in HC2.20 plants may be promoting a stronger selection pressure in favor of deleted virus variants.

\section{Recombination events observed in PVX-HC $\Delta$ variants.}

Sequences corresponding to $28 \mathrm{PVX}-\mathrm{HC} \Delta$ variants obtained from $\mathrm{HC} 2.6$ and $\mathrm{HCn} 11.20$ plants and to five obtained from $\mathrm{wt}$ $N$. benthamiana and HC2.20 plants were analyzed in order to identify conserved traits that could be informative about the recombination mechanisms that have led to deletion of HC-Pro sequences. These sequences were aligned to the original PVXHCT sequence as depicted in Figure 3. We observed that, in 17 of these 33 sequences, up to four consecutive nucleotides at
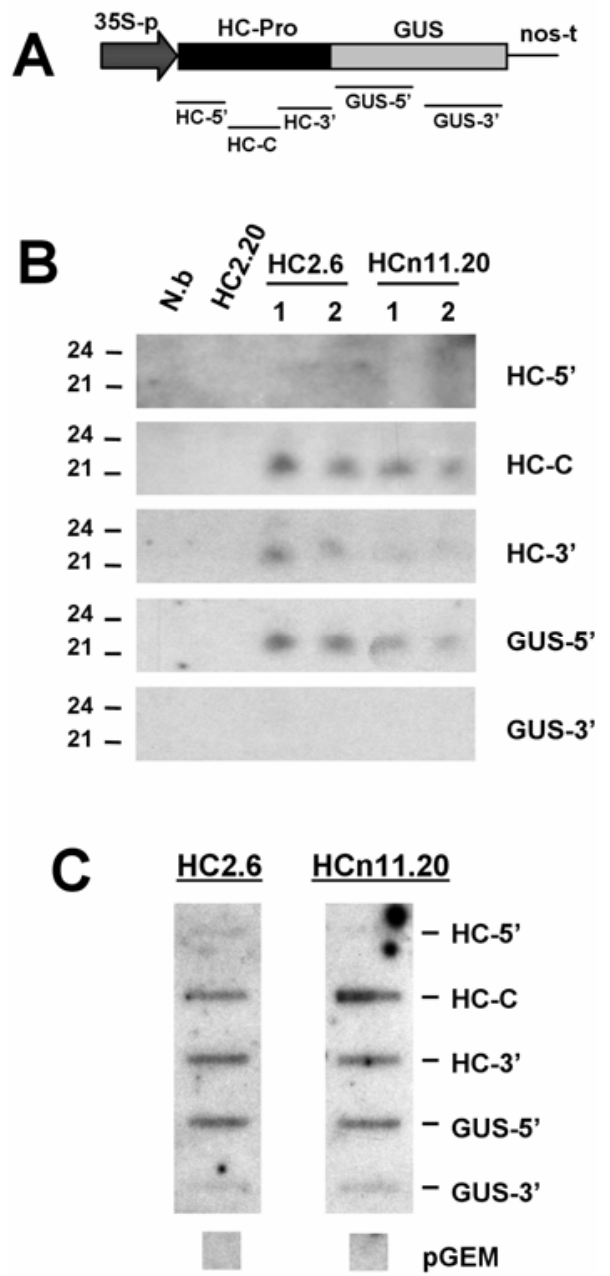

Fig. 4. Analysis of accumulation of siRNAs derived from different regions of the helper-component proteinase (HC-Pro) transgene. A, Diagram of the HC-Pro transgene construct showing the different probes used. B, Detection of siRNAs with labeled probes corresponding to the five different portions of the HC-Pro transgene. Two HC2.6 and two HCn11.20 plants were used together with a wild-type Nicotiana benthamiana plant (N.b) and an HC2.20 plant. Position of RNA markers (nt) are indicated on the left. C, Slot-blot hybridization of labeled siRNAs with DNA fragments corresponding to the five portions of the HC-Pro transgene. The plasmid pGEM was used as negative control of hybridization. one terminus of the aligned sequence overlapped with those in the deleted sequence immediately preceding the other terminus (Fig. 6A). The observed frequency of overlapping nucleotides was higher than that expected in a random situation (Fig. 6B), indicating an association of this trait with the recombination process. A second characteristic observed was a predominance of $\mathrm{A}$ and $\mathrm{T}$ among these overlapping nucleotides, as indicated in Figure 6C. Alignment of the sequences surrounding the deletion borders revealed other areas of moderate sequence similarity, indicating that sequence similarity may have assisted some of the recombination events.

\section{DISCUSSION}

We have shown that transgenic $N$. benthamiana plants containing a silenced HC-Pro transgene that promotes complete resistance against the homologous PPV were not completely resistant to a chimeric PVX containing the PPV HC-Pro gene. PVX-HCT escaped virus resistance promoted by the silenced HC-Pro transgene after removal of most of the targeted HCPro sequence. Once a critical amount of silencing-targeted sequence was lost, the deleted virus versions infected the plants systemically. The difference between PPV and PVX-HCT is obvious; although they both carry the same silencing-targeted sequence, PPV cannot lose the HC-Pro sequence without severely affecting its viability and, therefore, it cannot escape silencing-mediated resistance. Deletion of portions of the HCPro sequence in PVX-HCT is likely the result of RNA recombination processes occurring during virus replication (Nagy and Simon 1997). Consequently, the silenced HC-Pro plants used in this study must tolerate a minimum level of PVX-HCT replication to allow recombination to occur, even though PVXHCT is targeted by silencing. Our impression is that silencing promoted by the HC-Pro transgene in these plants is relatively weak (as judged by the relatively low amounts of siRNAs produced) and probably permits some initial rounds of replication, as observed in other silenced systems (English et al. 1996). In contrast, transgenic plants expressing an inverted repeat construct derived from Pepper mild mottle virus (PMMoV) (which produces high levels of siRNAs) were completely resistant to a chimeric PVX carrying the cognate PMMoV sequence, and no deleted PVX variants arose in these plants (M. Vargas, F. Tenllado, and J. R. Díaz-Ruíz, unpublished results).

We have observed that silencing of the HC-Pro transgene predominantly affects the central area of the transgene sequence with strikingly similar patterns in lines HC2.6 and HCn11.20. Previous studies have reported that silencing is frequently targeted predominantly against sequences corresponding to the $3^{\prime}$ area of transgenes (English et al. 1996; Sijen et al. 1996; Sonoda et al. 1999). However, this is not the only possible situation, as it has been observed in other studies (Hutvagner et al. 2000). The different patterns of siRNA production and target specificity observed in different studies probably reflect the way in which dsRNA from the transgene is produced in each system. This is likely conditioned by specific characteristics of the transgene sequence, transgene configuration, sites of integration into the chromosome, or a combination of these factors (Muskens et al. 2000). The length of sequence identity between the silenced transgene and the virus required to obtain efficient resistance against infection has also been investigated previously, using vectors that carried different portions and lengths of sequence identity with the transgene (English et al. 1996; Sijen et al. 1996; Sonoda et al. 1999). In general, the efficiency of resistance decreased with the length of the sequence shared by the virus and the silenced transgene.

In our study, we have taken advantage of a different approach; instead of designing PVX constructs with different target 
lengths, we have analyzed the PVX-HC $\Delta$ forms arising in silenced plants. It is conceivable that all PVX-HC $\Delta$ forms that accumulated systemically in silenced plants have lost enough silencing-targeted sequence as to surpass the selective filter imposed by silencing and, therefore, they were not efficient targets of the silencing machinery. The length of HC-Pro sequences retained in PVX-HCA variants differed, depending on the region of HC-Pro considered. Up to 526 nucleotides from the 5' region of HC-Pro was tolerated in PVX-HC $\Delta-10$, while only a maximum of 140 nucleotides was permitted from the $3^{\prime}$ region of HC-Pro (PVX-HC $\Delta-5)$. This fact agrees with the relative amounts of siRNAs produced from different regions of the HC-Pro transgene. For this reason, we should not consider the minimum amount of sequence identity required for efficient silencing in terms of length but in terms of the amount of siRNAs covering the targeted sequence. It could then be expected that in silenced systems producing high levels of siRNAs (e.g., plants transformed with inverted repeat constructs), a shorter length of sequence similarity is sufficient to promote efficient virus resistance. This is relevant when designing transgene constructs aimed to promote resistance against several different viruses (Prins 2003). In this way, smaller portions of sequence similarity could be used and resistance against more viruses might be attained in a simpler construct.

Recombination in RNA viruses is a widespread phenomenon that has a significant importance in virus evolution (Bonnet et al. 2005; García-Arenal et al. 2003). In most cases, observation of a recombinant virus arising in an infection process depends on its relative fitness compared with the parental virus. Therefore, detection of new recombinants relies on the application of selective pressures against the parental virus or in favor of the recombinants (Wintermantel and Schoelz 1996). Foreign inserts in virus vectors are likely a disadvantageous load for the virus and tend to be lost through recombination events as a result of selection pressure. Previous studies with PVX vectors have suggested a predilection for recombination between the two duplicated subgenomic promoter sequences, although other recombination events were also observed (Chapman et al. 1992). Also, TMV-based vectors seemed to have a more marked predilection for homologous recombination between duplicated sequences (Dawson et al. 1989). In our system, recombination events did not occur preferentially between identical sequences of the duplicated subgenomic promoters in the PVX vector, suggesting that long traces of sequence homology were not crucial in directing the recombination process. However, we observed short stretches of sequence similarity and a
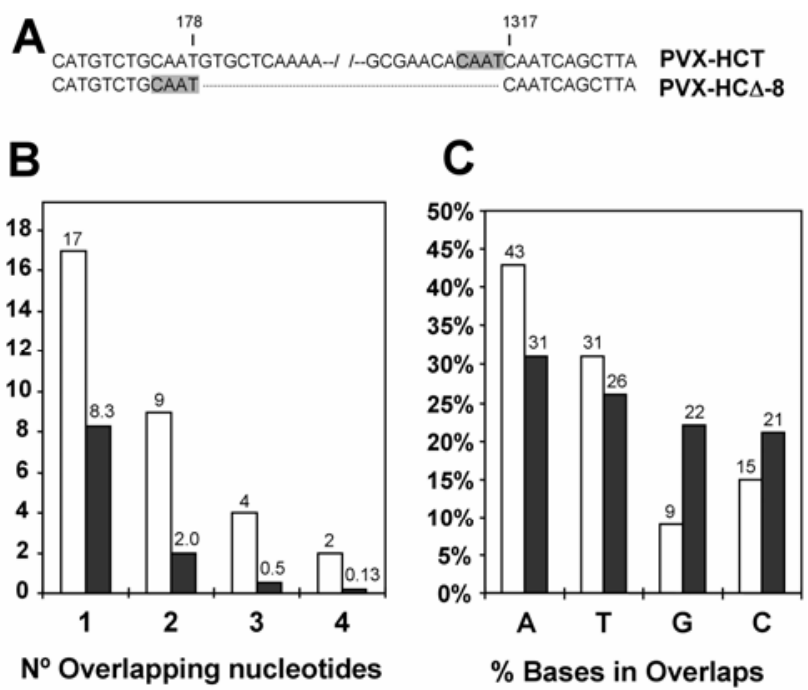

Fig. 6. Analysis of recombination processes in Potato virus $X(\mathrm{PVX})-\mathrm{HC} \Delta$ variants. A, Alignment of PVX-HC $\Delta-8$ and the original PVX-HCT. Coincident nucleotides at the junction site are shaded in gray. B, Number of observed coincidences affecting $1,2,3$, or 4 consecutive nucleotides at the junction sites (white bars). Number of expected coincidences for 1 nucleotide $(1 / 4$ of 33 sequences $=8.3), 2$ nucleotides $(1 / 16$ of $33=2.0), 3$ nucleotides $(1 / 64$ of $33=0.5)$, and 4 nucleotides $(1 / 256$ of $33=0.13)$ are indicated with dark bars. C, Frequency of A, T, G, or C among overlapping nucleotides at junction sites (white bars), compared with observed frequency of nucleotides throughout the helper-component proteinase sequence (dark bars).
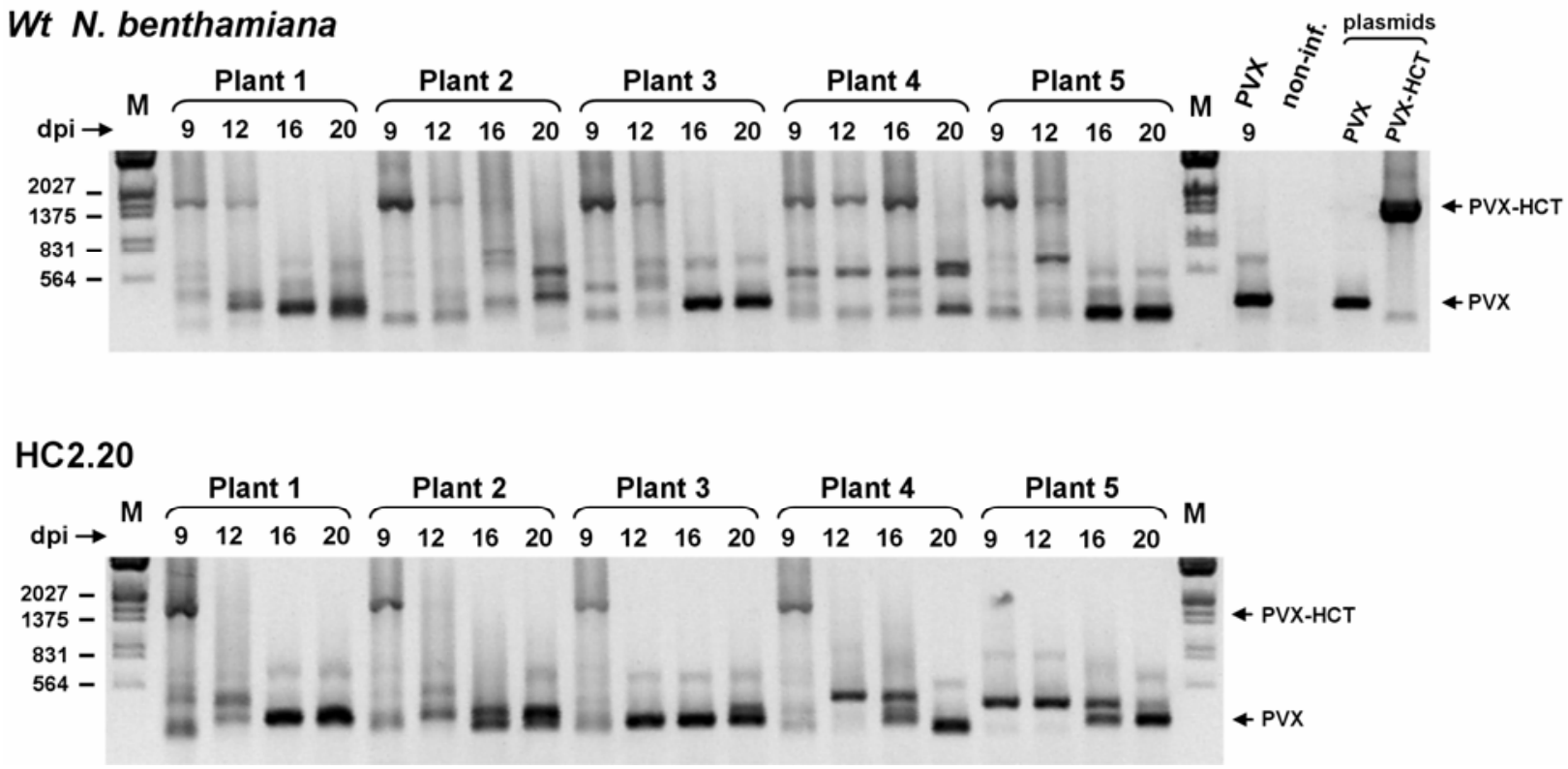

Fig. 5. Analysis of virus insert instability in wild-type (wt) and nonsilenced helper-component proteinase (HC-Pro) transgenic plants. Reverse transcriptasepolymerase chain reaction analysis of Potato virus $X(\mathrm{PVX})-\mathrm{HCL}_{134} \mathrm{H}$ instability in individual wt Nicotiana benthamiana and $\mathrm{HC} 2.20$ plants at 9 , 12,16 , and 20 days postinoculation. A plant infected with the empty PVX vector, a noninfected plant, and plasmids corresponding to PVX vector and PVX-HCT were used as size controls. The positions of PVX and PVX-HCT amplification products are indicated on the right of each panel. The positions of DNA markers (M) are indicated on the left. 
relative richness in $\mathrm{A}$ and $\mathrm{T}$ around the junction points, in accordance to what has been reported in previous studies (Guo et al. 1998; Mueller and Wimmer 1998). RNA recombination is thought to occur as a result of a copy-choice mechanism during replication (Cheng and Nagy 2003; Nagy and Simon 1997). The complex formed by the replicase and the nascent RNA is thought to dissociate from the RNA template and resume polymerization using a different RNA or a different part of the same RNA molecule as template. One of the factors suggested to favor the reassociation of the replicase-nascent RNA complex and the new RNA template, called 'acceptor strand,' is the sequence complementarity between the nascent and acceptor RNAs (Nagy and Simon 1997). Our results are in agreement with this model and suggest a moderate contribution of short stretches of sequence complementarity between the nascent and acceptor RNAs in driving the recombination process.

As indicated by our data, RNA silencing did not seem necessary to promote PVX-HCT recombination, as recombinant virus variants were observed in silenced HC2.6 and HCn11.20 plants, nonsilenced HC2.20 plants and in wt $N$. benthamiana plants. Therefore, the most likely scenario is that recombinant variants appear independently of the silencing status of the plant and that RNA silencing acts as a selective filter that accelerates the overtaking of the plant by specific virus variants. Consequently, in $\mathrm{HC} 2.6$ and $\mathrm{HCn} 11.20$ plants, in which silencing is likely to impose a strong selective filter, the original virus had already been outcompeted by recombinant variants in the second systemically infected leaf, while in HC2.20 and wt $N$. benthamiana, recombinant variants appeared and became dominant more slowly during the progress of systemic infection (typically in the third and fifth systemic leaves, respectively). In addition to this, a closer comparison of data from these HC2.20 and wt plants revealed that recombinant variants seemed to outcompete the original virus faster in HC2.20 than in wt $N$. benthamiana plants, suggesting that there is a higher selection pressure in favor of recombinant variants in HC2.20 plants. These plants contain a nonsilenced HC-Pro transgene that is likely targeted by VIGS during infection. In this process, the HC-Pro transgene may contribute to amplification of the RNA silencing induced by the virus and promote a stronger selection pressure in favor of recombinants with deletions in the HC-Pro insert.

Our results are in contrast with a recent report by Zhong and associates (2005) in which a TBSV vector retained a foreign GFP insert better in transgenic plants carrying a homologous GFP transgene than in wt $N$. benthamiana. Since RNA silencing induced by the virus affected the homologous transgene, it was suggested that targeting of the RNA silencing process against the foreign insert in the virus could compromise recombination by as-yet-unidentified mechanisms (Zhong et al. 2005). The difference between our results in HC2.6 and HCn11.20 plants and the results reported by Zhong and associates may arise from the fact that our plants were already silenced when the virus was introduced, whereas in the study by Zhong and associates, silencing was induced by the virus. However, our experimental set-up with HC2.20 and wt $N$. benthamiana plants was similar to that used by Zhong and associates, yet our results were opposite. Therefore, other differences such as specific properties of the viruses used or the kind of inserts might account for the discrepancies observed between these studies.

In our system, RNA silencing did not seem to negatively affect the recombination process. Even more, a possible role of silencing in promoting it should not be ignored. It has been suggested that the presence of truncated RNA templates may promote recombination by forcing replicase pause and dissociation when reaching the $5^{\prime}$ end of the truncated template (Cheng et al. 2006; Nagy and Simon 1997; White and Morris
1995). In a silenced system such as our HC-Pro transgenic plants, silencing targeted against the HC-Pro sequence in PVX-HCT may give rise to truncated viral RNAs that might promote recombination. This hypothesis should be considered when assessing the environmental risks of virus-resistant transgenic plants, because an input of virus that is targeted by silencing may give rise to truncated viral RNAs prone to undergo recombination with other viruses present in the plants. Nevertheless, the hypothetical risk of new virus varieties arising from the use of virus-resistant transgenic plants should always be compared with the already existent risk of recombination between two viruses in nontransgenic plants (Tepfer 2002).

\section{MATERIALS AND METHODS}

\section{Transgenic plants.}

$\mathrm{HC} 2.6$ and $\mathrm{HC} 2.20$ are $\mathrm{R}_{2}$ lines progeny of HC2 plants described previously (Barajas et al. 2004). These lines contain a transgene construct carrying the HC-Pro gene sequence from PPV isolate 5.15 (López-Moya et al. 1994). The HC-Pro transgene in $\mathrm{HC} 2.6$ plants is silenced and the plants are resistant to PPV, while in HC2.20 plants the HC-Pro transgene is not silenced and the plants are susceptible to PPV. HCn11.20 is a PPV-resistant $\mathrm{R}_{2}$ line carrying a silenced nontranslatable $\mathrm{HC}$ Pro transgene. This line was obtained in the same manner as HC lines (Barajas et al. 2004), while introducing a start codon followed by an in-frame stop codon at the beginning of the HC-Pro sequence. $\mathrm{C} 2.2$ is an $\mathrm{R}_{2}$ line transformed with the empty vector $\mathrm{pBI} 121$. All plants were maintained in a growth chamber at 22 to $25^{\circ} \mathrm{C}$ with a 16-h light and 8-h dark cycle.

\section{Plasmid constructs.}

Plasmids PVX-HCT (PVX-HCwt) and PVX-HCL ${ }_{134} \mathrm{H}$ have been described previously (González-Jara et al. 2005). They are based on plasmid pP2C2S-402, a cDNA clone of PVX (strain UK-3) derived from pP2C2S (Baulcombe et al. 1995). PVX-HCT contains a functional, translatable HC-Pro version, while PVX-HCL ${ }_{134} \mathrm{H}$ contains an $\mathrm{HC}$-Pro version with a leucine to histidine mutation in amino acid 134 that makes it nonfunctional in suppression of gene silencing. To prepare plasmids PVX-HC $\Delta-5$, PVX-HC $\Delta-6$, PVX-HC $\Delta-10$, and PVX-HC $\Delta-2 \mathrm{c}$, the PVX region surrounding the HC-Pro insertion site was amplified by RT-PCR from the corresponding infected plants. These PCR products were digested with EagI and XmaI to excise the remaining HC-Pro portions, which were cloned into similarly digested $\mathrm{pP} 2 \mathrm{C} 2 \mathrm{~S}-402$. To prepare PVX-HC-5', PVXHC-C, and PVX-HC-3', HC-Pro portions corresponding to the $5^{\prime}$ region (nucleotides 1 to 443 of PPV HC-Pro sequence; Laín et al. 1989), central region (nucleotides 453 to 895), and $3^{\prime}$ region (nucleotides 866 to 1,374 ), respectively, were amplified with $P f u$ polymerase and were cloned into EcoRV-digested pP2C2S-402.

\section{In vitro transcriptions and virus inoculations.}

PVX derived plasmids were linearized with SpeI or SphI and were purified by phenol/chloroform extraction and ethanol precipitation. Approximately $120 \mathrm{ng}$ of linearized plasmid were used per plant to produce infective transcripts. Transcription reactions included $1 \times$ transcription buffer (Roche, Branchburg, NJ, U.S.A.), $0.25 \mathrm{mM}$ ATP, CTP, and UTP, 0.025 $\mathrm{mM}$ GTP, $0.5 \mathrm{mM} \mathrm{m}^{7} \mathrm{G}\left(5^{\prime}\right) \mathrm{ppp}\left(5^{\prime}\right) \mathrm{G}$ (cap analog, Roche), $1 \mathrm{U}$ of RNasin (Promega, Madison, WI. U.S.A.) per microliter, and $1 \mathrm{U}$ of RNA polymerase T7 (Roche) per microliter. These reactions were incubated for 15 min for cap incorporation and, afterwards, were supplemented with $0.25 \mathrm{mM}$ GTP and incubated for $1 \mathrm{~h}$. Plants were inoculated by applying $5 \mu \mathrm{l}$ of the transcription reaction per plant onto celite-dusted leaves. 
To prepare PVX-HCT sap inocula, we collected the first leaves showing systemic symptoms from infected plants and ground these in 10 volumes of $20 \mathrm{mM}$ sodium phosphate buffer ( $\mathrm{pH}$ 7.0). This $1 \times$ inoculum was subsequently diluted in sodium phosphate buffer as required. Inoculation of plants was performed by applying $20 \mu \mathrm{l}$ of sap inoculum onto Carborundum-dusted leaves. PPV sap inoculum was prepared as described previously (Barajas et al. 2004).

\section{RNA extraction and analysis.}

Samples for virus analysis were taken from systemically infected leaves. Leaf tissue $(100 \mathrm{mg}$ ) was ground in $500 \mu \mathrm{l}$ of Trizol reagent (Invitrogen, San Diego, CA, U.S.A.). The aqueous phase was separated by adding $100 \mu \mathrm{l}$ of chloroform, RNA was precipitated with 1 volume of isopropanol, and the pellet was washed in $70 \%$ ethanol. The RNA pellet was air-dried and resuspended in RNase-free water. Total RNA ( $2 \mu \mathrm{g})$ was used for Northern blot analysis of PVX-HCT-derived viruses, using digoxigenin (DIG)-labeled RNA probes (DIG RNA labeling kit; Roche) complementary to the PVX CP gene or to the PPV HC-Pro gene as described (González-Jara et al. 2005).

For RT-PCR analysis of virus stability, RT reactions included $0.5 \mu \mathrm{g}$ of total RNA, $1 \mu \mathrm{M}$ reverse primer, $1 \times \mathrm{RT}$ buffer (Promega), $0.4 \mathrm{mM}$ each dNTP, $1 \mathrm{U}$ of RNasin (Promega) per microliter, and $0.15 \mathrm{U}$ AMV reverse transcriptase (Promega) per microliter. RNA and primer were first incubated $5 \mathrm{~min}$ at $65^{\circ} \mathrm{C}$. Afterwards, they were placed at $43^{\circ} \mathrm{C}$, the rest of the components were added, and then, they were incubated for 2 h. PCR reactions were performed with Taq polymerase using 2 $\mu \mathrm{l}$ of RT reactions as template in $25 \mu \mathrm{l}$ of final volume. For analysis of PVX-HCT stability in HC2.6 and HCn11.20 plants (Fig. 3A), RT was performed with primer AAAGAATTCGGT GGTAGAGTGACAAC (complementary to position 6,445 to 6,429 of PVX vector). For PCR primers, CATGCCATGGATAT TCTCATCAGTAG and TTTAAGCTTGTTCCAGTGATACGAC (corresponding to positions 4,486 to 4,505 and 6,414 to 6,397 of PVX vector, respectively) were used. For analysis of PVX$\mathrm{HCL}_{134} \mathrm{H}$ stability in wt $N$. benthamiana and $\mathrm{HC} 2.20$ plants (Fig. 5), RT was performed with primer GTTGCGCCTGCAG TTTTTG (complementary to position 5,812 to 5,794 of PVX vector), and PCR with primers AATACATATCTCAACGCAATC and TGGTAGTTGAGGTAGTTGA (corresponding to positions 5,436 to 5,456 and 5,793 to 5,775 of PVX vector, respecttively).

Detection of siRNAs derived from the HC-Pro transgene was performed as previously described (Barajas et al. 2004), using ${ }^{32} \mathrm{P}$-labeled probes corresponding to transgene portions HC-5', HC-C, HC-3' (nucleotides 1 to 443, 453 to 895, and 866 to 1,374 of HC-Pro sequence, respectively), GUS-5', and GUS-3' (nucleotides 13 to 860 and 968 to 1,804 of GUS sequence, respectively).

For slot-blot analysis of siRNA accumulation, total RNA was extracted with Trizol, and 1 to $2 \mathrm{mg}$ resolved in polyacrylamide-urea gels. A gel slice containing 15- to 35-nt RNAs was excised and cut into pieces. RNA was then eluted from the sliced gel in $80 \%$ formamide, $40 \mathrm{mM}$ PIPES, pH 6.4, $1 \mathrm{mM}$ EDTA, $400 \mathrm{mM} \mathrm{NaCl}$ (Hutvagner et al. 2000) and was subsequently precipitated with 2.5 volumes of ethanol, $0.3 \mathrm{M}$ sodium acetate, and $0.05 \mu \mathrm{g}$ of glycogen per microliter, and was washed in $70 \%$ ethanol, air dried, and resuspended in RNasefree water. Resulting RNA ( 2 to $3 \mu \mathrm{g}$ ) was dephosphorylated with calf intestine phosphatase and purified with phenol/chloroform extraction and ethanol precipitation. Dephosphorylated siRNA fraction 300 (ng) was labeled with T4 polynucleotide kinase and $50 \mu \mathrm{Ci}$ of $\gamma{ }^{-}{ }^{3} \mathrm{P}$-ATP and was then used as probe for slot-blot hybridization in PerfectHyb Plus Buffer (Sigma, St. Louis) at $38^{\circ} \mathrm{C}$. Slot blots were prepared applying $50 \mathrm{ng}$ of purified DNA per slot, using a Bio-Dot SF apparatus (BioRad, Hertfordshire, U.K.), following the manufacturer's instructions.

\section{AKNOWLEDGMENTS}

D. Barajas was the recipient of a doctoral fellowship from the Spanish Ministry of Education and Science (MEC). This study was supported by grants BIO2003-03428 from Comision Interministerial de Ciencia y Tecnologia (CICYT)-MEC, GR/SAL/0831/2004 from Comunidad de Madrid, and 200540M109 from Consejo Superior De Investigaciones Cientificas (CSIC)-Comunidad de Madrid (Spain).

\section{LITERATURE CITED}

Barajas, D., Tenllado, F., González-Jara, P., Martínez-García, B., Atencio, F. A., and Díaz-Ruíz, J. R. 2004. Resistance to Plum pox virus (PPV) in Nicotiana benthamiana plants transformed with the PPV HC-Pro silencing suppressor gene. J. Plant Pathol. 86:239-248.

Baulcombe, D. 2004. RNA silencing in plants. Nature 431:356-363.

Baulcombe, D. C., Chapman, S., and Santa-Cruz, S. 1995. Jellyfish green fluorescent protein as a reporter for virus infections. Plant J. 7:10451053.

Beachy, R. N. 1997. Mechanisms and applications of pathogen-derived resistance in transgenic plants. Curr. Opin. Biotechnol. 8:215-220.

Bonnet, J., Fraile, A., Sacristan, S., Malpica, J. M., and García-Arenal, F. 2005. Role of recombination in the evolution of natural populations of Cucumber mosaic virus, a tripartite RNA plant virus. Virology 332:359368.

Burch-Smith, T. M., Anderson, J. C., Martin, G. B., and Dinesh-Kumar, S. P. 2004. Applications and advantages of virus-induced gene silencing for gene function studies in plants. Plant J. 39:734-746.

Cañizares, M. C., Nicholson, L., and Lomonossoff, G. P. 2005. Use of viral vectors for vaccine production in plants. Immunol. Cell Biol. 83:263-270.

Chapman, S., Kavanagh, T., and Baulcombe, D. 1992. Potato virus X as a vector for gene expression in plants. Plant J. 2:549-557.

Chapman, E. J., Prokhnevsky, A. I., Gopinath, K., Dolja, V. V., and Carrington, J. C. 2004. Viral RNA silencing suppressors inhibit the microRNA pathway at an intermediate step. Genes Dev. 18:1179-1186.

Cheng, C. P., and Nagy, P. D. 2003. Mechanism of RNA recombination in carmo- and tombusviruses: Evidence for template switching by the RNA-dependent RNA polymerase in vitro. J. Virol. 77:12033-12047.

Cheng, C. P., Serviene, E., and Nagy, P. D. 2006. Suppression of viral RNA recombination by a host exoribonuclease. J. Virol. 80:2631-2640.

Dawson, W. O., Lewandowski, D. J., Hilf, M. E., Bubrick, P., Raffo, A. J., Shaw, J. J., Grantham, G. L., and Desjardins, P. R. 1989. A tobacco mosaic virus-hybrid expresses and loses an added gene. Virology 172:285292.

Dunoyer, P., Lecellier, C. H., Parizotto, E. A., Himber, C., and Voinnet, O. 2004. Probing the microRNA and small interfering RNA pathways with virus-encoded suppressors of RNA silencing. Plant Cell 16:1235-1250.

English, J. J., Mueller, E., and Baulcombe, D. C. 1996. Suppression of virus accumulation in transgenic plants exhibiting silencing of nuclear genes. Plant Cell 8:179-188.

García-Arenal, F., Fraile, A., and Malpica, J. M. 2003. Variation and evolution of plant virus populations. Int. Microbiol. 6:225-232.

González-Jara, P., Atencio, F. A., Martínez-García, B., Barajas, D., Tenllado, F., and Díaz-Ruíz, J. R. 2005. A single nucleotide mutation in the Plum pox virus HC-Pro gene abolishes both synergistic and silencing suppression activities. Phytopathology 95:894-901.

Guo, H. S., López-Moya, J. J., and García, J. A. 1998. Susceptibility to recombination rearrangements of a chimeric plum pox potyvirus genome after insertion of a foreign gene. Virus Res. 57:183-195.

Hutvagner, G., Mlynarova, L., and Nap, J. P. 2000. Detailed characterization of the posttranscriptional gene-silencing-related small RNA in a GUS gene-silenced tobacco. RNA 6:1445-1454.

Lai, M. M. 1992. RNA recombination in animal and plant viruses. Microbiol. Rev. 56:61-79.

Laín, S., Riechmann, J. L., and García, J. A. 1989. The complete nucleotide sequence of plum pox potyvirus RNA. Virus Res. 13:157-172.

Llave, C., Tenllado, F., and Díaz-Ruíz, J. R. 2004. RNA silencing and other small RNA-related processes as innovative molecular tools for control of virus diseases in plants. Pages 271-298 in: Emerging concepts in plant health management. R. T. Lartey and A. J. Caesar (eds). Research Signpost, Kerala, India.

López-Moya, J. J., Canto, T., López-Abella, D., and Díaz-Ruíz, J. R. 1994. Differentiation of Mediterranean Plum pox virus isolates by coat protein analysis. Plant Pathol. 43:164-171. 
Moissiard, G., and Voinnet, O. 2004. Viral suppression of RNA silencing in plants. Mol. Plant Pathol. 5:71-82.

Mueller, S., and Wimmer, E. 1998. Expression of foreign proteins by poliovirus polyprotein fusion: Analysis of genetic stability reveals rapid deletions and formation of cardioviruslike open reading frames. J. Virol. 72:20-31.

Muskens, M. W., Vissers, A. P., Mol, J. N., and Kooter, J. M. 2000. Role of inverted DNA repeats in transcriptional and post-transcriptional gene silencing. Plant Mol. Biol. 43:243-260.

Nagy, P. D., and Simon, A. E. 1997. New insights into the mechanisms of RNA recombination. Virology 235:1-9.

Prins, M. 2003. Broad virus resistance in transgenic plants. Trends Biotechnol. 21:373-375.

Rabindran, S., and Dawson, W. O. 2001. Assessment of recombinants that arise from the use of a TMV-based transient expression vector. Virology 284:182-189.

Scholthof, H. B., Scholthof, K. B., and Jackson, A. O. 1996. Plant virus gene vectors for transient expression of foreign proteins in plants. Annu. Rev. Phytopathol. 34:299-323.

Sijen, T., Wellink, J., Hiriart, J. B., and Van Kammen, A. B. 1996. RNAmediated virus resistance: Role of repeated transgenes and delineation of targeted regions. Plant Cell 8:2277-2294.

Silhavy, D., and Burgyan, J. 2004. Effects and side-effects of viral RNA silencing suppressors on short RNAs. Trends Plant Sci. 9:76-83.

Sonoda, S., Mori, M., and Nishiguchi, M. 1999. Homology-dependent virus resistance in transgenic plants with the coat protein gene of sweet potato feathery mottle potyvirus: Target specificity and transgene methylation. Phytopathology 89:385-391.
Szittya, G., Molnar, A., Silhavy, D., Hornyik, C., and Burgyan, J. 2002. Short defective interfering RNAs of tombusviruses are not targeted but trigger post-transcriptional gene silencing against their helper virus. Plant Cell 14:359-372.

Tenllado, F., and Díaz-Ruíz, J. R. 1999. Complete resistance to pepper mild mottle tobamovirus mediated by viral replicase sequences partially depends on transgene homozygosity and is based on a gene silencing mechanism. Transgenic Res. 8:83-93.

Tenllado, F., Llave, C., and Díaz-Ruíz, J. R. 2004. RNA interference as a new biotechnological tool for the control of virus diseases in plants. Virus Res. 102:85-96.

Tepfer, M. 2002. Risk assessment of virus-resistant transgenic plants. Annu. Rev. Phytopathol. 40:467-491.

Voinnet, O. 2005. Induction and suppression of RNA silencing: Insights from viral infections. Nat. Rev. Genet. 6:206-220.

White, K. A., and Morris, T. J. 1995. RNA determinants of junction site selection in RNA virus recombinants and defective interfering RNAs. RNA 1:1029-1040.

Wintermantel, W. M., and Schoelz, J. E. 1996. Isolation of recombinant viruses between Cauliflower mosaic virus and a viral gene in transgenic plants under conditions of moderate selection pressure. Virology 223:156-164.

Zamore, P. D., and Haley, B. 2005. Ribo-gnome: The big world of small RNAs. Science 309:1519-1524.

Zhong, X., Hou, H., and Qiu, W. 2005. Integrity of nonviral fragments in recombinant Tomato bushy stunt virus and defective interfering RNA is influenced by silencing and the type of inserts. Mol. Plant-Microbe Interact. 18:800-807. 